\title{
Correlation Between Changes in the Serum Magnesium Concentration and Visceral Fat Volume in Patients With Type 2 Diabetes Receiving Luseogliflozin: A Sub-Analysis of Data From the LIGHT Study
}

\author{
Tatsuo Yanagawa ${ }^{\mathrm{a}, \mathrm{b}}$, Hideshi Matsuda ${ }^{\mathrm{a}}$, Masahiro Sugawara ${ }^{\mathrm{c}}$, \\ Masahiro Fukuda $^{\mathrm{d}}$, Takashi Sasakie, f, g
}

\begin{abstract}
Background: Sodium-glucose cotransporter 2 (SGLT-2) inhibitors have been reported to more effectively suppress cardiovascular events (CVEs) in patients with type 2 diabetes. The underlying mechanism, however, remains unknown. SGLT-2 inhibitors have the unique beneficial effect of ameliorating hypomagnesemia, which is a well-known independent risk factor for CVE. However, the mechanism by which SGLT-2 inhibitors increase the serum magnesium $(\mathrm{Mg})$ levels also remains unknown. We hypothesized that SGLT-2 inhibitor-induced visceral fat loss might also be correlated with the serum $\mathrm{Mg}$ levels.
\end{abstract}

Methods: We conducted a sub-analysis of the data of 31 patients with type 2 diabetes who were treated with luseogliflozin in the LIGHT study. The correlations between changes in the serum $\mathrm{Mg}$ concentration $(\Delta \mathrm{Mg})$ and the baseline patient characteristics/changes in the values of clinical parameters at 24 weeks were analyzed by multiple regression analysis.

Results: The $\Delta \mathrm{Mg}$ was significantly associated with the baseline serum $\mathrm{Mg}$ concentration ( $\beta=-0.47,95 \%$ confidence interval $(\mathrm{CI}):-0.84$ $-0.13 ; \mathrm{P}=0.01)$ and $\Delta$ visceral fat volume $(\mathrm{VFV})(\beta=-0.33,-0.59$ $-0.09 ; \mathrm{P}=0.03)$.

Conclusions: We found that elevation of serum $\mathrm{Mg}$ concentrations after SGLT-2 inhibitor treatment was associated with two factors; low serum $\mathrm{Mg}$ concentrations before the start of treatment and decrease in

Manuscript submitted April 5, 2021, accepted April 20, 2021

Published online April 25, 2021

aDepartment of Medicine, Nerima General Hospital, Tokyo, Japan

b Institute of Healthcare Quality Improvement, Public Interest Incorporated Foundation, Tokyo Healthcare Foundation, Tokyo, Japan

'Sugawara Clinic, Nerima, Tokyo, Japan

${ }^{\mathrm{d}}$ Fukuda Clinic, Osaka, Japan

Institute of Clinical Medicine and Research, Research Center for Medical Sciences, The Jikei University School of Medicine, Chiba, Japan

fSasaki Institute, Sasaki Foundation, Tokyo, Japan

'Corresponding Author: Takashi Sasaki, Sasaki Institute, Sasaki Foundation,

2-2 Kanda-Surugadai, Chiyoda-ku, Tokyo 101-0062, Japan.

Email: tsasaki-endo@umin.ac.jp

doi: https://doi.org/10.14740/jem738 the VFV after treatment.

Keywords: Type 2 diabetes; SGLT-2 inhibitors; Visceral fat; Magnesium; Cardiovascular events; Metabolic syndrome; Obesity

\section{Introduction}

Reducing the risk of cardiovascular events (CVEs) is a key goal in the treatment of type 2 diabetes mellitus, although even large studies, including the UKPDS [1], have failed to clearly establish that conventional metabolic control can decrease the risk of CVE [2-4]. Since 2007, the Food and Drug Administration (FDA) has mandated non-inferiority testing; that is, after adjusting for existing risk factors such as hemoglobin Alc (HbA1c), blood pressure, lipid profile, and smoking status, it must be demonstrated that newly developed antidiabetic drugs do not increase the risk of CVE as compared to existing drugs. Unlike other oral antidiabetic drugs, sodium-glucose cotransporter 2 (SGLT-2) inhibitors have been shown to suppress CVE $[5,6]$. According to these studies, SGLT-2 inhibitors appear to decrease the risk of CVE even from the early stages of treatment. Since the effects of metabolic improvement would be expected to take several years to appear, this observation cannot be explained by the effects of metabolic improvement alone. Although various hypotheses have been proposed [7], there is so far no widely accepted consensus on the underlying mechanism.

SGLT-2 inhibitors have the unique effect of improving hypomagnesemia [8]. Magnesium (Mg) is a coenzyme for various enzymes involved in both insulin secretion and sensitivity [9], and hypomagnesemia has been shown to be associated with both the development of diabetes mellitus and obesity. In addition, because $\mathrm{Mg}$ has anti-inflammatory, antisympathetic, and antiarrhythmic effects [9], Mg deficiency is also a risk factor for the development of CVE; and correction of the serum $\mathrm{Mg}$ concentration has been shown to reduce CVE and sudden death [10-12]. Systemic Mg depletion is common in obese and diabetic patients, and is often overlooked because the serum concentrations are often within the reference range [9]. Based 
on the above findings, we hypothesized that SGLT-2 inhibitors suppress the risk of CVE by improving hypomagnesemia $[13,14]$. The exact mechanism by which SGLT-2 inhibitors increase serum $\mathrm{Mg}$ concentrations remains unknown [15]. Insufficient glycemic control may increase the urinary $\mathrm{Mg}$ excretion, leading to hypomagnesemia [16], and SGLT-2 inhibitors may suppress urinary $\mathrm{Mg}$ excretion, thereby correcting the serum $\mathrm{Mg}$ levels. However, while there are no data yet to lend support to this hypothesis, negative data have also been reported [17]. Therefore, we proposed a different hypothesis from the effect of the drug on the urinary $\mathrm{Mg}$ excretion.

It is well known that hypomagnesemia associated with obesity can be ameliorated by reducing the weight by dieting or bariatric surgery [18]. In addition, reduction of the visceral fat volume (VFV) by bariatric surgery has been shown to be correlated with increased serum Mg concentrations [19]. Therefore, we hypothesized that reduction of the VFV by any means, even other than by bariatric surgery, could increase the serum Mg levels. To verify this hypothesis, data on the VFV and serum $\mathrm{Mg}$ concentration would be needed, and these data had already been collected in the LIGHT study (Luseogliflozin: the Components of Weight Loss in Japanese Patients with Type 2 Diabetes Mellitus) [20], in which SGLT-2 inhibitors were demonstrated to reduce the VFV. We attempted to determine the correlation between the serum Mg levels and VFV reduction by conducting a sub-analysis of the data from the LIGHT study.

\section{Materials and Methods}

Since the methodology is described in detail in the LIGHT study [20], we shall only present an outline here. Patients recruited for this study were between 20 and 65 years of age, and had type 2 diabetes mellitus, with glycated hemoglobin (HbA1c) levels in the range of 7.0-10.5\%, and body mass index (BMI) values in the range of 20.0 - 35.0.

The patients were treated through the study period according to the standard diet therapy protocol indicated by the Japan Diabetes Society [21]. After the period of observation, SGLT-2 inhibitor treatment was added-on for those with poor glycemic control. The patients received $2.5 \mathrm{mg}$ of luseogliflozin once daily, in the morning, with an optional titration up of the dose to $5 \mathrm{mg}$ once daily from week 12 or later, in case of insufficient efficacy. No patients are taking drugs such as proton pump inhibitors (PPIs) and diuretics that change serum $\mathrm{Mg}$ levels.

\section{Evaluated variables and the evaluation time-points}

In the LIGHT study, the patients were followed up for up to 52 weeks, but in this sub-analysis, we conducted the evaluation using data measured at 24 weeks. Since there was no significant change in metabolism or fat mass from 24 weeks to 52 weeks, we thought that it was appropriate to use the data measured at 24 weeks. BMI was calculated as follows: weight (in kilograms) divided by height (in meters) squared. The fasting plasma glucose (FPG), HbA1c (National Glycohemoglobin
Standardization Program (NGSP)), estimated glomerular filtration rate (eGFR), serum levels of adiponectin (ADP), triglyceride (TG), high-density lipoprotein cholesterol (HDL-C), $\mathrm{Mg}$, calcium $(\mathrm{Ca})$, potassium $(\mathrm{K})$, sodium $(\mathrm{Na})$, and phosphate $(\mathrm{P})$, and the subcutaneous fat volume (SFV) and VFV were measured at the start of the treatment as well as at the end of 24 weeks of treatment.

The values at 24 weeks minus the values at the baseline are expressed as $\Delta$ values (e.g., $\Delta \mathrm{Mg}=(\mathrm{Mg}$ concentration at 24 weeks) - (Mg concentration at the baseline)). All variables were collected from all 31 patients before and after treatment.

\section{Laboratory methods}

The laboratory tests were outsourced to LSI Medience Corporation (Tokyo, Japan). The body components were measured using whole-body dual-energy X-ray absorptiometry (DXA; Hologic/Discovery, Hologic/Delphi; Hologic Inc., Marlborough, MA, USA; or GE/Lunar Prodigy enCORE 2006; GE Healthcare, Madison, WI, USA). The VFV and SFV were determined in coronal computed tomographic images of the abdomen at the level of the umbilicus. Evaluation of the abdominal computed tomographic images was carried out at a designated institution by a single predefined radiologist. The SFV and VFV were analyzed using Slim Vision (CYBERNET SYSTEMS CO., LTD., Tokyo, Japan).

\section{Statistical analysis}

For analysis of the changes in individual data, the last observation carried forward imputation method was applied. Data are presented as means \pm standard deviation (SD). Differences in the variables between the groups were compared using the Student's $t$-test for paired data. When the data were not normally distributed, they were shown by interquartile range (IQR) and the Wilcoxon singed-rank test was used.

To investigate the correlations between the $\Delta \mathrm{Mg}$ and other variables, Pearson's correlation coefficient test was used. When the data were not normally distributed, Spearman's rank correlation coefficient was calculated. Multiple linear regression analysis (forced-entry method) was used to identify factors that were significantly associated with the $\Delta \mathrm{Mg}$. The statistical analyses were performed using the SPSS software v25. $\mathrm{P}<0.05$ was set as being indicative of statistical significance.

\section{Institutional review board approval}

The study protocol was reviewed and approved by the Institutional Review Board of the Jikei University School of Medicine on January 15, 2015 (approval number: 26-278) and the Institutional Review Board of Nerima General Hospital on February 17, 2015 (approval number: 20150217-1). The study was registered in advance at the University Hospital Medical Information Network Clinical Trials Registry (UMIN000015112). 
Table 1. Clinical Characteristics at the Baseline and at 24 Weeks After the Administration of Luseogliflozin

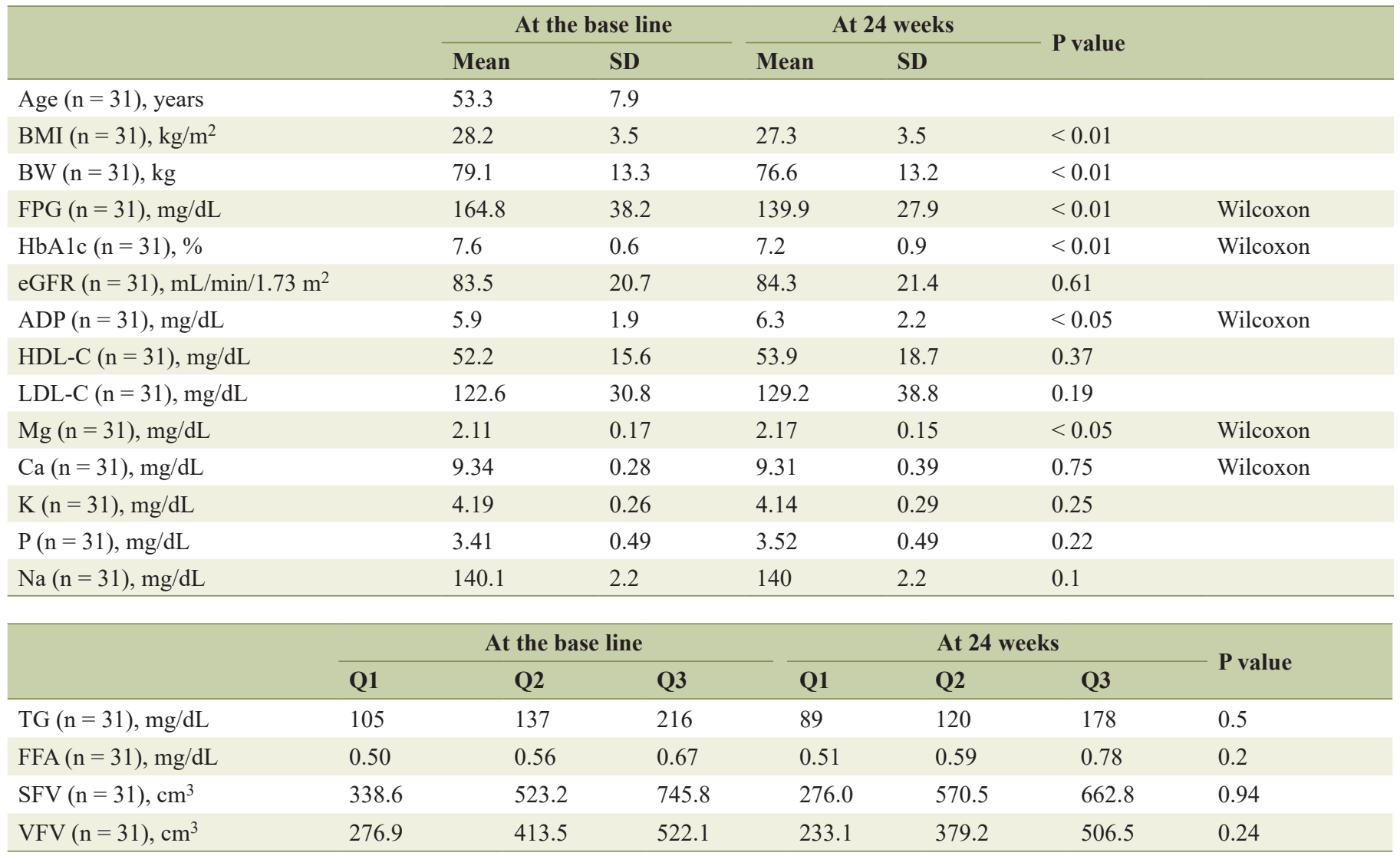

Data are presented as means \pm standard deviation (SD). Differences in the variables between the groups were compared using the Student's $t$-test for paired data. When the data were not normally distributed, they were shown by interquartile range (IQR) and the Wilcoxon singed-rank test was used. BMl: body mass index; BW: body weight; FPG: fasting plasma glucose; HbA1c (National Glycohemoglobin Standardization Program (NGSP)): hemoglobin A1C; eGFR: estimated glomerular filtration rate, ADP: adiponectin; HDL-C: high-density lipoprotein cholesterol; LDL-C: low-density lipoprotein cholesterol; TG: triglyceride; FFA: free fatty acid; SFV: subcutaneous fat volume; VFV: visceral fat volume; Mg: magnesium; Ca: calcium; K: potassium; P: phosphate; Na: sodium.

\section{Ethical considerations}

The present study was carried out in compliance with the Declaration of Helsinki, and the ethical guideline for clinical research published by the Ministry of Health, Labor and Welfare, Japan.

\section{Results}

\section{Clinical characteristics at the baseline and after 24 weeks of luseogliflozin treatment}

As shown in Table 1, the data of 31 Japanese patients with type 2 diabetes were analyzed in this study, after excluding six of the 37 patients included in the LIGHT study (data on the $\mathrm{Mg}$ concentrations were not available in two patients, and data on the fat areas were not available in the remaining four patients). The study cohort consisted of 22 men and nine women with a mean age of $53.3 \pm 7.9$ years. Clinical param- eters evaluated prior to the start of luseogliflozin treatment are summarized in Table 1. After 24 weeks of luseogliflozin treatment, a significant increase of the serum $\mathrm{Mg}$ concentration, and improvement of the BMI, body weight (BW), FPG, HbAlc, and serum ADP level were observed. While the VFV was found to be significantly reduced in the LIGHT study, the reduction of the VFV did not reach statistical significance in this sub-analysis, presumably because six cases were excluded for the reasons described above.

\section{Analysis of the correlations of the $\Delta \mathrm{Mg}$ with various clini- cal parameters}

To identify factors influencing the changes in the serum $\mathrm{Mg}$ concentrations after 24 weeks of treatment, analysis of the correlations between the $\Delta \mathrm{Mg}$ and various parameters is shown in Table 2 . The $\Delta \mathrm{Mg}$ was significantly correlated with the baseline serum $\mathrm{Mg}$ concentration $(\mathrm{r}=-0.77, \mathrm{P}<0.01), \Delta \mathrm{HbAlc}$ $(\mathrm{r}=-0.42, \mathrm{P}<0.05), \Delta \mathrm{VFV}(\mathrm{r}=-0.37, \mathrm{P}<0.05)$, and $\Delta \mathrm{P}(\mathrm{r}=$ $-0.37, \mathrm{P}<0.05)$. No statistically significant changes of any of 
Table 2. Analysis of the Correlations of $\Delta \mathrm{Mg}$ With Various Parameters

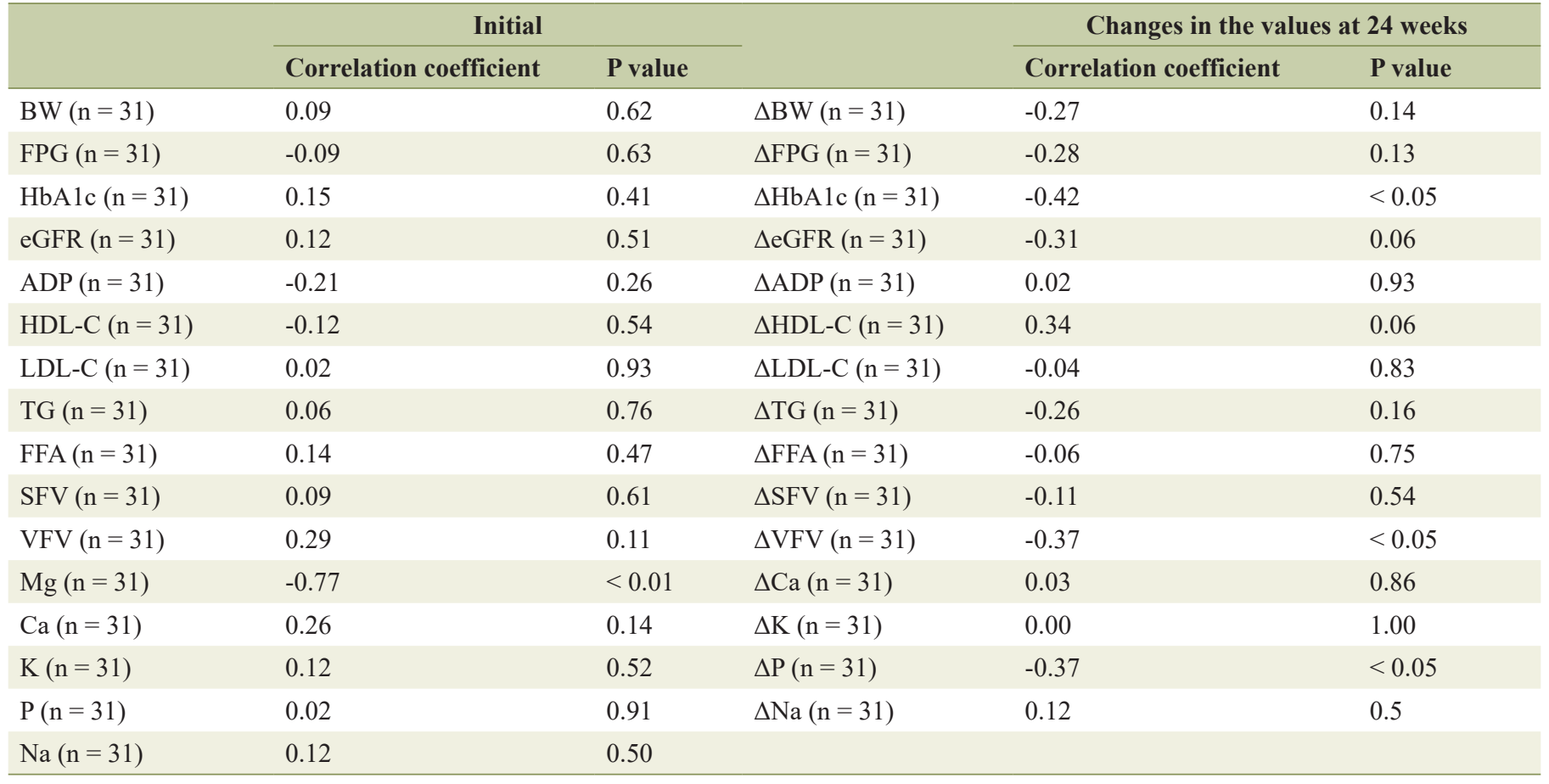

The data at 24 weeks minus the data at the baseline are expressed as $\Delta$ (e.g., $\Delta \mathrm{Mg}=(\mathrm{Mg}$ concentration at 24 weeks) - (Mg concentration at the baseline)). BW: body weight; FPG: fasting plasma glucose; HbA1c (National Glycohemoglobin Standardization Program (NGSP)): hemoglobin A1c; eGFR: estimated glomerular filtration rate; ADP: adiponectin; HDL-C: high-density lipoprotein cholesterol; LDL-C: low-density lipoprotein cholesterol; TG: triglyceride; FFA: free fatty acid; SFV: subcutaneous fat volume; VFV: visceral fat volume; Mg: magnesium; Ca: calcium; K: potassium; P: phosphate; Na: sodium.

the other variables were observed.

\section{Multivariate linear regression analysis}

To identify the determinants of the $\Delta \mathrm{Mg}$ after 24 weeks of luseogliflozin treatment, multiple regression analysis (forcedentry method) was performed.

The baseline serum $\mathrm{Mg}, \Delta \mathrm{HbA} 1 \mathrm{c}, \Delta \mathrm{VFV}$ and $\Delta \mathrm{P}$ were regarded as independent variables, since all of these variables were significantly correlated with the $\Delta \mathrm{Mg}$, as shown in Table 2. Furthermore, BMI, age, and gender were considered to be important factors and were added as independent variables. The results are shown in Table 3 . The $\Delta \mathrm{Mg}$ was significantly associated with the baseline serum $\mathrm{Mg}$ concentration $(\beta=$ $-0.47,95 \%$ confidence interval $(\mathrm{CI}):-0.84--0.13 ; \mathrm{P}=0.01)$ and $\triangle \operatorname{VFV}(\beta=-0.33,-0.59--0.09 ; \mathrm{P}=0.03)$. The other five variables did not contribute significantly to changes in serum Mg levels.

\section{Discussion}

Hypomagnesemia is often observed in patients with diabetes mellitus and obesity [9]. Hypomagnesemia is known as a risk factor for acute myocardial infarction, exacerbation of heart failure, fatal arrhythmias, and sudden death $[10,11]$. It has also been reported that a Mg-rich diet and correction of hypomagnesemia reduce the risk of CVE [12]. Since SGLT-2 inhibitors are known to correct hypomagnesemia $[8,15]$, it would be reasonable to hypothesize that decrease the risk of CVE by SGLT- 2 inhibitors is mediated by elevated serum $\mathrm{Mg}$ levels $[13,14]$. However, the mechanism by which SGLT-2 inhibitors increase the serum $\mathrm{Mg}$ concentrations remains unclear $[8,15]$. This study was conducted with the objective of elucidating the underlying mechanism.

In this analysis, SGLT-2 inhibitor-induced elevation of the serum $\mathrm{Mg}$ concentrations were found to be significantly correlated with the decreased VFV observed after treatment, as well as low serum Mg concentrations at the study baseline. The former is found in all SGLT-2 inhibitors, the latter in at least canagliflozin [22]. Therefore, this effect is considered to be common not only to luseogliflozin but also to other SGLT-2 inhibitors. These results will be discussed in order below.

\section{Correlation between increase of the serum Mg concentra- tions and decrease of the VFV}

In this study, we report for the first time that increased serum $\mathrm{Mg}$ levels observed following SGLT-2 inhibitor therapy were associated with a decreased VFV. This correlation between $\mathrm{VFV}$ and serum $\mathrm{Mg}$ levels is not unique to patients receiving 
Table 3. The Determinants of the $\Delta \mathrm{Mg}$ After 24 Weeks of Luseogliflozin Treatment

\begin{tabular}{llll} 
& $\boldsymbol{\beta}$ & $\mathbf{9 5 \%}$ CI & P value \\
\hline Baseline serum Mg concentrations & -0.47 & $-0.84,-0.13$ & 0.01 \\
$\Delta \mathrm{VFV}$ & -0.33 & $-0.59,-0.09$ & 0.03 \\
$\Delta \mathrm{HbA1}$ & -0.24 & $-0.46,0.03$ & 0.15 \\
$\Delta \mathrm{P}$ & -0.15 & $-0.36,0.09$ & 0.36 \\
BMI & 0.12 & $-0.12,0.32$ & 0.45 \\
Age & -0.11 & $-0.31,0.11$ & 0.47 \\
Sex & 0.04 & $-0.09,0.13$ & 0.76 \\
\hline
\end{tabular}

To identify the determinants of the $\Delta \mathrm{Mg}$ after 24 weeks of luseogliflozin treatment, multiple regression analysis (forced-entry method) was performed. The data at 24 weeks minus the data at the baseline are expressed as $\Delta$ (e.g., $\Delta \mathrm{Mg}=$ (Mg concentration at 24 weeks) - (Mg concentration at the baseline)). Mg: magnesium; VFV: visceral fat volume; HbA1c (NGSP): hemoglobin A1c; P: phosphate; BMI: body mass index; Cl: confidence interval.

SGLT-2 inhibitor therapy. Dietary weight reduction is known to be associated with elevated serum Mg levels [18]. Decrease of the VFV following bariatric surgery is also reported to be correlated with an increase of the serum Mg levels [19]. Based on these findings, together with the findings of this sub-analysis, we believe that reduction of the VFV by any method (dietary/surgical) is likely to be associated with increase of the serum $\mathrm{Mg}$ concentrations.

Serum Mg levels are regulated by renal excretion, absorption from the intestinal tract, and intracellular and extracellular redistribution [9]. So far, little has been discussed about the relationship between the serum $\mathrm{Mg}$ concentrations and the VFV. In obese individuals, excess visceral fat induces insulin resistance, which results in hypomagnesemia due to the greater need for intracellular $\mathrm{Mg}$, which is a coenzyme for enzymes involved in insulin secretion and sensitivity. When insulin resistance is alleviated by weight reduction, the intracellular need for $\mathrm{Mg}$ is reduced, and $\mathrm{Mg}$ is redistributed extracellularly with increase of the serum $\mathrm{Mg}$ concentrations. Alternatively, we hypothesize that the increased binding of $\mathrm{Mg}$ to the visceral fat in association with increased VFV results in lower serum Mg levels. There is as yet no evidence that $\mathrm{Mg}$ binds to visceral fat, but such binding is considered plausible, because $\mathrm{Mg}$ binds directly to plasma non-esterified fatty acids (NEFA) resulting in reduced plasma $\mathrm{Mg}$ concentrations [23].

\section{The lower the serum Mg concentration before the start of treatment, the greater the degree of increase of the serum Mg concentration after SGLT-2 inhibitor treatment}

This has already been reported by another group [22], although the underlying mechanism still remains to be identified. In the LIGHT study, we observed that the greater the VFV in the baseline, the greater the decrease in VFV accompanied by treatment with SGLT-2 inhibitor. Therefore, we thought that the low serum Mg level might be normalized proportional to the decrease in VFV.

Correction of hypomagnesemia is important, because hypomagnesemia is a strong risk factor for the development of CVE. As obesity predisposes to hypomagnesemia, obese individuals are probably the most likely to benefit from the CVE- reducing effect of SGLT-2 inhibitor treatment.

\section{Is it possible that SGLT-2 inhibitors increase serum Mg levels by reducing urinary $\mathrm{Mg}$ excretion?}

It has been suggested that poor glycemic control can lead to hypomagnesemia by increasing the urinary $\mathrm{Mg}$ excretion [16]. Therefore, SGLT-2 inhibitors may increase the serum $\mathrm{Mg}$ levels by reducing the urinary $\mathrm{Mg}$ excretion. However, there has been no investigation on the effect of SGLT-2 inhibitor treatment on the urinary $\mathrm{Mg}$ excretion since the 2009 report of elevation of the serum Mg concentration by SGLT-2 inhibitors $[8,14]$. Recently, it was reported that SGLT-2 inhibitor treatment exerted no effect on the urinary $\mathrm{Mg}$ excretion [17]. We have no data to prove in this regard. This is an issue to be solved in the future.

\section{Limitation of this study}

This is an uncontrolled study with limited power. Therefore, due to unidentifiable bias, nonspecific effects or regression to the normal range may be mistakenly shown as a result of analysis. However, in sub-analyzing the previous LIGHT study, an underlying study for present paper, we have found that there is an association between reduced VFV and recovery of serum $\mathrm{Mg}$ levels in patients with low serum $\mathrm{Mg}$. Moreover, the greater the VFV was in the baseline, the greater the decrease in VFV accompanied by treatment with SGLT-2 inhibitor. We consider that in order to understand the unknown mechanism for the recovery of $\mathrm{Mg}$, based on this observation, a more accurate elucidation should be conducted by a controlled trail using more cases.

In conclusion, the increase in the serum $\mathrm{Mg}$ concentrations observed following treatment with SGLT-2 inhibitors was strongly associated with low serum $\mathrm{Mg}$ concentrations at the baseline and the reduced VFV after treatment. In other words, the observed increase in the serum $\mathrm{Mg}$ concentrations after treatment with luseogliflozin can be explained by the decrease in the VFV observed after treatment, rather than by an inherent pharmacologic effect of SGLT-2 inhibitors. 


\section{Acknowledgments}

None to declare.

\section{Financial Disclosure}

This research received financial support from Taisho Toyama Pharmaceutical Co., Ltd. (T Sasaki). The sponsor had no role in the research design, data collection, data analysis, data interpretation, or report preparation.

\section{Conflict of Interest}

None to declare.

\section{Informed Consent}

Informed consent was obtained from each of the patients prior to their participation in this study.

\section{Author Contributions}

TS has decided on a research plan and protocol and amended the manuscript. HM, MF, and MS provided patient data. TY was a major contributor in writing the manuscript, analyzing and interpreting all the data. All authors read and approved the final manuscript.

\section{Data Availability}

The authors declare that data supporting the findings of this study are available within the article.

\section{References}

1. Intensive blood-glucose control with sulphonylureas or insulin compared with conventional treatment and risk of complications in patients with type 2 diabetes (UKPDS 33). UK Prospective Diabetes Study (UKPDS) Group. Lancet. 1998;352(9131):837-853.

2. Scirica BM, Bhatt DL, Braunwald E, Steg PG, Davidson J, Hirshberg B, Ohman P, et al. Saxagliptin and cardiovascular outcomes in patients with type 2 diabetes mellitus. N Engl J Med. 2013;369(14):1317-1326.

3. White WB, Cannon CP, Heller SR, Nissen SE, Bergenstal RM, Bakris GL, Perez AT, et al. Alogliptin after acute coronary syndrome in patients with type 2 diabetes. N Engl J Med. 2013;369(14):1327-1335.

4. Green JB, Bethel MA, Armstrong PW, Buse JB, Engel SS, Garg J, Josse R, et al. Effect of sitagliptin on cardiovascular outcomes in type 2 diabetes. N Engl J Med.
2015;373(3):232-242.

5. Zinman B, Wanner C, Lachin JM, Fitchett D, Bluhmki E, Hantel S, Mattheus M, et al. Empagliflozin, cardiovascular outcomes, and mortality in type 2 diabetes. N Engl J Med. 2015;373(22):2117-2128.

6. Neal B, Perkovic V, Mahaffey KW, de Zeeuw D, Fulcher G, Erondu N, Shaw W, et al. Canagliflozin and cardiovascular and renal events in type 2 diabetes. N Engl J Med. 2017;377(7):644-657.

7. Ferrannini E, Mark M, Mayoux E. CV Protection in the EMPA-REG OUTCOME trial: a "Thrifty Substrate" hypothesis. Diabetes Care. 2016;39(7):1108-1114.

8. List JF, Woo V, Morales E, Tang W, Fiedorek FT. Sodium-glucose cotransport inhibition with dapagliflozin in type 2 diabetes. Diabetes Care. 2009;32(4):650-657.

9. Grober U, Schmidt J, Kisters K. Magnesium in prevention and therapy. Nutrients. 2015;7(9):8199-8226.

10. Qu X, Jin F, Hao Y, Li H, Tang T, Wang H, Yan W, et al. Magnesium and the risk of cardiovascular events: a meta-analysis of prospective cohort studies. PLoS One. 2013;8(3):e57720.

11. Tangvoraphonkchai K, Davenport A. Magnesium and cardiovascular disease. Adv Chronic Kidney Dis. 2018;25(3):251-260

12. Del Gobbo LC, Imamura $\mathrm{F}, \mathrm{Wu} \mathrm{JH}$, de Oliveira Otto MC, Chiuve SE, Mozaffarian D. Circulating and dietary magnesium and risk of cardiovascular disease: a systematic review and meta-analysis of prospective studies. Am J Clin Nutr. 2013;98(1):160-173.

13. Yanagawa T. Is an increase in serum magnesium one of the causes of cardiovascular events reduction in the EMPAREG OUTCOME study? J Clin Med Res. 2017;9(5):449450.

14. Ray EC. Evolving understanding of cardiovascular protection by SGLT2 inhibitors: focus on renal protection, myocardial effects, uric acid, and magnesium balance. Curr Opin Pharmacol. 2020;54:11-17.

15. Tang H, Zhang X, Zhang J, Li Y, Del Gobbo LC, Zhai $\mathrm{S}$, Song Y. Elevated serum magnesium associated with SGLT2 inhibitor use in type 2 diabetes patients: a metaanalysis of randomised controlled trials. Diabetologia. 2016;59(12):2546-2551.

16. Gommers LM, Hoenderop JG, Bindels RJ, de Baaij JH. Hypomagnesemia in Type 2 Diabetes: A Vicious Circle? Diabetes. 2016;65(1):3-13.

17. Solini A, Giannini L, Seghieri M, Vitolo E, Taddei S, Ghiadoni L, Bruno RM. Dapagliflozin acutely improves endothelial dysfunction, reduces aortic stiffness and renal resistive index in type 2 diabetic patients: a pilot study. Cardiovasc Diabetol. 2017;16(1):138.

18. Mikalsen SM, Bjorke-Monsen AL, Whist JE, Aaseth J. Improved magnesium levels in morbidly obese diabetic and non-diabetic patients after modest weight loss. Biol Trace Elem Res. 2019;188(1):45-51.

19. Johansson HE, Zethelius B, Ohrvall M, Sundbom M, Haenni A. Serum magnesium status after gastric bypass surgery in obesity. Obes Surg. 2009;19(9):1250-1255.

20. Sasaki T, Sugawara M, Fukuda M. Sodium-glucose cotransporter 2 inhibitor-induced changes in body compo- 
sition and simultaneous changes in metabolic profile: 52 week prospective LIGHT (Luseogliflozin: the Components of Weight Loss in Japanese Patients with Type 2 Diabetes Mellitus) Study. J Diabetes Investig. 2019;10(1):108-117.

21. Food Exchange Lists - Dietary guidance for persons with diabetes. Japan Diabetes Society. 2012.

22. Gilbert RE, Mende C, Vijapurkar U, Sha S, Davies MJ, Desai M. Effects of canagliflozin on serum magnesium in patients with type 2 diabetes mellitus: a post hoc analysis of randomized controlled trials. Diabetes Ther. 2017;8(2):451-458.

23. Kurstjens S, de Baaij JHF, Overmars-Bos C, van den Munckhof ICL, Garzero V, de Vries MA, Burggraaf B, et al. Increased NEFA levels reduce blood $\mathrm{Mg}(2+)$ in hypertriacylglycerolaemic states via direct binding of NEFA to $\operatorname{Mg}(2)$. Diabetologia. 2019;62(2):311-321. 